\title{
Characteristics of colistin-resistant Escherichia coli from pig farms in Central China
}

\author{
Zhong Peng ${ }^{1,2,3,4+} \mathbb{D}$, Xiaoxue Zhang ${ }^{1,2,3,4+}$, Xiaosong Li, ${ }^{1,2,3,4}$, Zizhe Hu${ }^{1,2,3,4}$, Zugang Li ${ }^{1,2,3,4}$, Chaoying Jia ${ }^{1,2,3,4}$, \\ Menghong Dai ${ }^{2,3,4}$, Chen Tan ${ }^{1,2,3,4}$, Huanchun Chen ${ }^{1,2,3,4}$ and Xiangru Wang ${ }^{1,2,3,4^{*}}$ (i)
}

\begin{abstract}
The emergence and dissemination of colistin resistance in Enterobacteriaceae mediated by plasmid-borne mcr genes in recent years now pose a threat to public health. In this study, we isolated and characterized colistinresistant and/or mcr-positive E. coli from pig farms in Central China. Between 2018 and 2019, 594 samples were collected and recovered $445 \mathrm{E}$. coli isolates. Among them, 33 with colistin resistance phenotypes and 37 that were positive for $m c r$ genes were identified, including 34 positive for $m c r-1$, one positive for $m c r-3$, and two positive for both mcr-1 and mcr-3. An insertion of nine bases ("CTGGATACG") into mcr-1 in four mcr-positive isolates led to gene dysfunction, and therefore did not confer the colistin resistance phenotype. Antimicrobial susceptibility testing revealed that $37 \mathrm{mcr}$-positive isolates showed severe drug resistance profiles, as 50\% of them were resistant to 20 types of antibiotics. Multilocus sequence typing revealed a heterogeneous group of sequence types in mcr-positive isolates, among which ST10 (5/37), ST156 (5/37), and ST617 (4/37) were the predominant types. Plasmid conjugation assays showed that $\mathrm{mcr}$-carrying plasmids of $25 \mathrm{mcr}$-positive isolates were conjugated with $E$. coli recipient, with conjugation frequencies ranging from $1.7 \times 10^{-6}$ to $4.1 \times 10^{-3}$ per recipient. Conjugation of these $\mathrm{mcr}$ genes conferred a colistin resistance phenotype upon the recipient bacterium. PCR typing of plasmids harbored in the 25 transconjugants determined six types of plasmid replicons, including IncX4 (14/25), FrepB (4/25), Incl2 (3/25), IncHI2 (2/25), FIB (1/25), and Incl1 (1/25). This study contributes to the current understanding of antibiotic resistance and molecular characteristics of colistin-resistant $E$. coli in pig farms.
\end{abstract}

Keywords: Escherichia coli, Colistin resistance, mcr positivity, Antimicrobial resistance, Sequence types, Plasmid types, Plasmid conjugation

\footnotetext{
*Correspondence: wangxr228@mail.hzau.edu.cn

${ }^{\dagger}$ Zhong Peng and Xiaoxue Zhang contributed equally to this work.

${ }^{1}$ State Key Laboratory of Agricultural Microbiology, College of Veterinary

Medicine, Huazhong Agricultural University, No.1 Shizishan Street, Hongshan

District, Wuhan, China

${ }^{2}$ Key Laboratory of Preventive Veterinary Medicine in Hubei Province, The

Cooperative Innovation Center for Sustainable Pig Production, Huazhong

Agricultural University, Wuhan, China

Full list of author information is available at the end of the article
}

(c) The Author(s). 2021, corrected publication 2021. Open Access This article is licensed under a Creative Commons Attribution 4.0 International License, which permits use, sharing, adaptation, distribution and reproduction in any medium or format, as long as you give appropriate credit to the original author(s) and the source, provide a link to the Creative Commons licence, and indicate if changes were made. The images or other third party material in this article are included in the article's Creative Commons licence, unless indicated otherwise in a credit line to the material. If material is not included in the article's Creative Commons licence and your intended use is not permitted by statutory regulation or exceeds the permitted use, you will need to obtain permission directly from the copyright holder. To view a copy of this licence, visit http://creativecommons.org/ licenses/by/4.0/. The Creative Commons Public Domain Dedication waiver (http://creativecommons.org/publicdomain/zero/1.

0/) applies to the data made available in this article, unless otherwise stated in a credit line to the data. 


\section{Background}

Having first been discovered in the 1940s, polymyxins are an old family of chemically distinct lipopeptide antibiotics produced by the gram-positive bacterium Paenibacillus polymyxa (Li et al. 2019a). In recent years, with the rapid increase in multidrug-resistant gram-negative pathogens in the clinic, particularly the "superbugs" Acinetobacter baumannii, Pseudomonas aeruginosa, Klebsiella pneumoniae, and Escherichia coli, several polymyxin antibiotics, especially polymyxin $B$ and polymyxin $E$ (or colistin), have been used as the last therapeutic option for infections caused by these pathogens ( $\mathrm{Li}$ et al. 2019b). Mechanistically, polymyxin antibiotics largely exert their primary antimicrobial mode of action by permeabilizing bacterial outer membrane through a direct interaction with lipopolysaccharide ( $\mathrm{Li}$ et al. 2019c). However, bacteria have still successfully developed several resistance mechanisms to combat the effect of polymyxins (Wang et al. 2021). Of great concern is the mechanism conferred by plasmidmediated $m c r$ gene (Liu et al. 2016). $m c r$ gene encodes a phosphoethanolamine (PEtN) transferase that helps to add a PEtN moiety to lipid A of lipopolysaccharide, increasing its cationic charges and consequently decreasing the binding of colistin to lipopolysaccharide (El-Sayed Ahmed et al. 2020; Sun et al. 2018). Since the report about a plasmid carrying $m c r-1$ in $E$. coli from both humans and animals in China in 2016 (Liu et al. 2016), this colistin resistance mechanism has received extensive attention worldwide. In addition to $m c r-1$, nine plasmid-borne $\mathrm{mcr}$ genes $(m c r-2 \sim m c r-10)$ have been identified to date (Wang et al. 2020a). These $10 \mathrm{mcr}$ genes have been detected in bacterial isolates from humans, animals, foods of animal origin, and environment, and they confer resistance to polymyxins (Andrade et al. 2020). The emergence of these genes may accelerate global movement towards a postantibiotic era (Du et al. 2016; Paterson and Harris 2016). Therefore, it is of great importance to monitor the prevalence of $m c r$-bearing bacteria in clinical activities.

As the bacterium in which plasmid-carrying $\mathrm{mcr}$ gene was first determined (Liu et al. 2016), E. coli is an important zoonotic and foodborne pathogen that has a great capacity to accumulate resistance genes, mostly

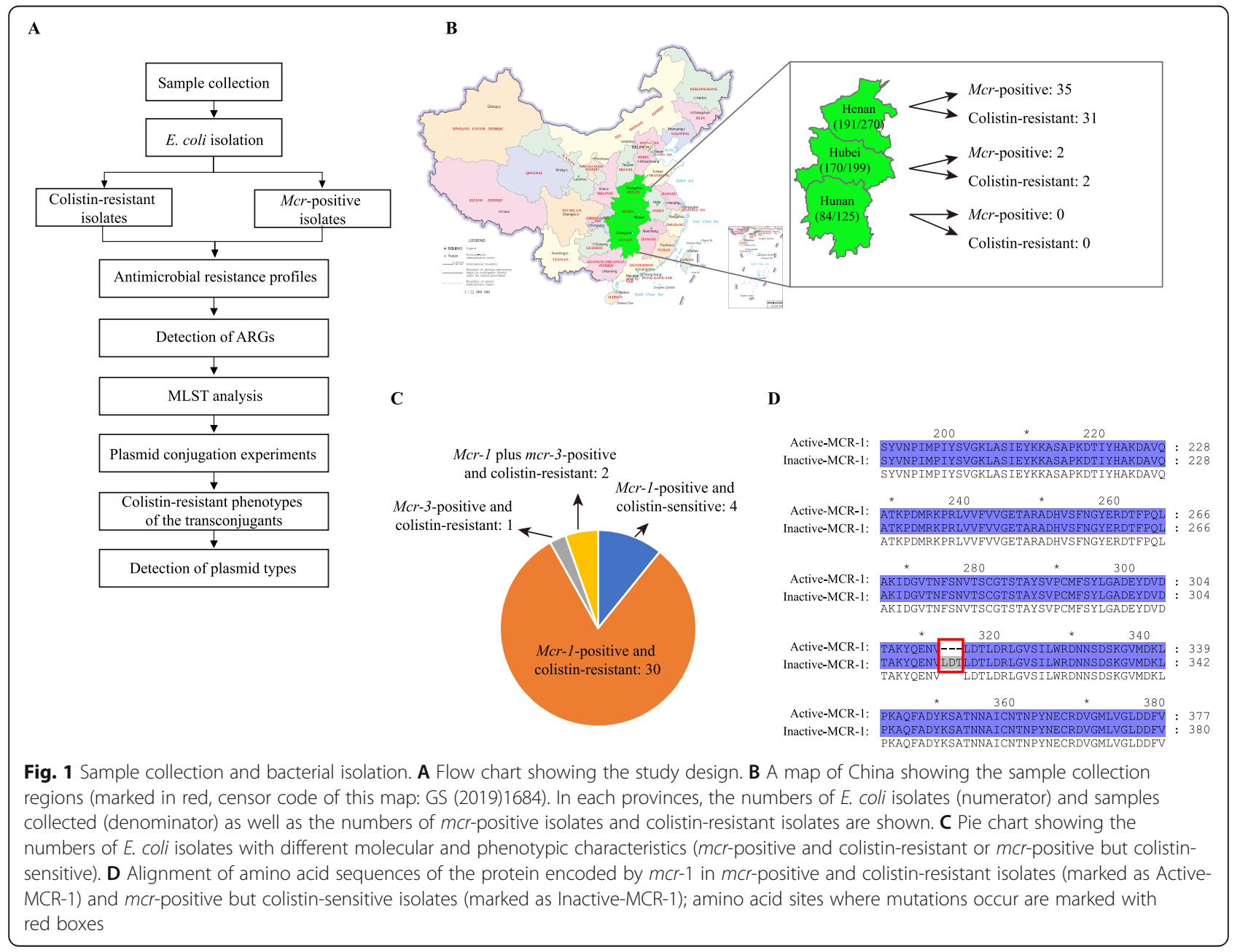


through horizontal gene transfer. This bacterial species is recognized as a "natural reservoir" of antimicrobial resistance genes (ARGs) (Poirel et al. 2018). It's also one of the most frequently recovered bacteria in livestock, in which the overuse and abuse of antimicrobials is proposed as a primary reason for the acceleration, development and spread of resistant bacteria (van Boeckel et al. 2017). From this point on, it will be necessary to monitor the epidemiological characteristics of drug-resistant $E$. coli in livestock. China is the largest pig-rearing country of the world. In this study, we report the isolation and antimicrobial resistance phenotypes as well as genetic characteristics of colistin-resistant $E$. coli and/or $m c r$-positive $E$. coli from pig farms in Central China, which is one of the primary pig-producing regions in China. The aim of this study is to reveal the current prevalence and diversion of colistin-resistant E. coli in Chinese pig farms.

\section{Results}

Isolation of colistin-resistant $E$. coli and mcr-positive $E$. coli from pig farms in Central China

By performing bacterial isolation and identification (Fig. 1A), 445 E. coli strains were recovered from 594 farm-origin samples in Central China between 2018 and 2019 (Fig. 1B). The total isolation rate was 74.9\%. Phenotype screening assays were performed in $33 \mathrm{E}$. coli isolates using agar containing $2 \mu \mathrm{g} / \mathrm{mL}$ colistin $(7.42 \%$; 33/
445). However, PCR detection identified 37 E. coli isolates were positive for $\mathrm{mcr}$ genes (8.31\%; 37/445), including 34 isolates positive only for $m c r-1$, one isolate positive only for $m c r-3$, and two isolates positive for both $m c r-1$ and mcr-3 (Fig. 1C). These 37 mcr-positive isolates included the 33 isolates determined by phenotype screening assay. Tests on MIC (minimum inhibitory concentration) of colistin among these $37 \mathrm{mcr}$-positive isolates revealed that MIC of 33 isolates ranging from $4 \mu \mathrm{g} / \mathrm{mL}$ to $8 \mu \mathrm{g} / \mathrm{mL}$, while the remaining four isolates lower than $0.5 \mu \mathrm{g} / \mathrm{mL}$ (Table 1).

To understand why the four mcr-1-carrying $E$. coli isolates were sensitive to colistin, $m c r-1$ genes were cloned from both colistin-resistant and colistin-sensitive isolates. Nucleotide sequencing and sequence alignments revealed that $m c r-1$ harbored by the four colistinsensitive $E$. coli had an insertion of nine bases (“CTGGATACG") at sites 946 954 bp compared to $m c r-1$ in colistin-positive isolates, which led to an insertion of three amino acid residues ("LDT") at sites 314 316 in mobile colistin resistance (MCR) protein as encoded by four colistin-sensitive $E$. coli (Fig. 1D). Next, $m c r-1$ genes harbored by colistin-resistant E. coli ( $m c r-$ $\left.1^{\mathrm{R}}\right)$ and colistin-sensitive E. coli $\left(m c r-1^{\mathrm{S}}\right)$ were cloned into the commercially available plasmid $\mathrm{pMD}^{\mathrm{m}} 19-\mathrm{T}$ (TAKARA, Japan). pMD19- $m c r-1^{\mathrm{R}}$, pMD19-mcr-1 ${ }^{\mathrm{S}}$, and pMD19-T were then transformed into TOP10 chemically competent E. coli (Thermo-Fisher, US).

Table 1 Minimum inhibitory concentration (MIC) values for colistin of 37 mcr-positive E. coli isolates

\begin{tabular}{|c|c|c|c|c|c|}
\hline Strain & Mcr profile & MIC $(\mu \mathrm{g} / \mathrm{mL})$ & Strain & Mcr profile & MIC $(\mu \mathrm{g} / \mathrm{mL})$ \\
\hline HeN1 & $m c r-1$ & 8 & HeN212 & $m c r-1$ & 4 \\
\hline HeN7 & $m c r-1$ & 8 & HeN219 & $m c r-1+m c r-3$ & 4 \\
\hline HeN12 & $m c r-1$ & 8 & HeN227 & $m c r-3$ & 4 \\
\hline HeN2O & $m c r-1$ & 4 & HeN228 & $m c r-1+m c r-3$ & 4 \\
\hline $\mathrm{HeN} 24$ & $m c r-1$ & 4 & HeN229 & $m c r-1$ & 4 \\
\hline HeN33 & $m c r-1$ & 4 & HeN241 & $m c r-1$ & 4 \\
\hline HeN35 & $m c r-1$ & 4 & HeN249 & $m c r-1$ & 4 \\
\hline HeN98 & $m c r-1$ & 4 & HeN252 & $m c r-1$ & 4 \\
\hline HeN100 & $m c r-1$ & 4 & HeN253 & $m c r-1$ & 4 \\
\hline HeN115 & $m c r-1$ & 4 & HeN257 & $m c r-1$ & 4 \\
\hline HeN191 & $m c r-1$ & 4 & HeN261 & $m c r-1$ & 4 \\
\hline HeN192 & $m c r-1$ & 4 & HeN267 & $m c r-1$ & 4 \\
\hline HeN194 & $m c r-1$ & 4 & HeN268 & $m c r-1$ & 4 \\
\hline HeN195 & $m c r-1$ & 4 & HuB15 & $m c r-1$ & 4 \\
\hline HeN198 & $m c r-1$ & 4 & HuB54 & $m c r-1$ & 4 \\
\hline HeN199 & $m c r-1$ & 4 & HeN86 & $m c r-1$ & $<0.25$ \\
\hline HeN206 & $m c r-1$ & 4 & HeN87 & $m c r-1$ & $<0.5$ \\
\hline \multirow[t]{2}{*}{ HeN208 } & $m c r-1$ & 4 & HeN88 & $m c r-1$ & $<0.25$ \\
\hline & & & HeN204 & $m c r-1$ & $<0.5$ \\
\hline
\end{tabular}




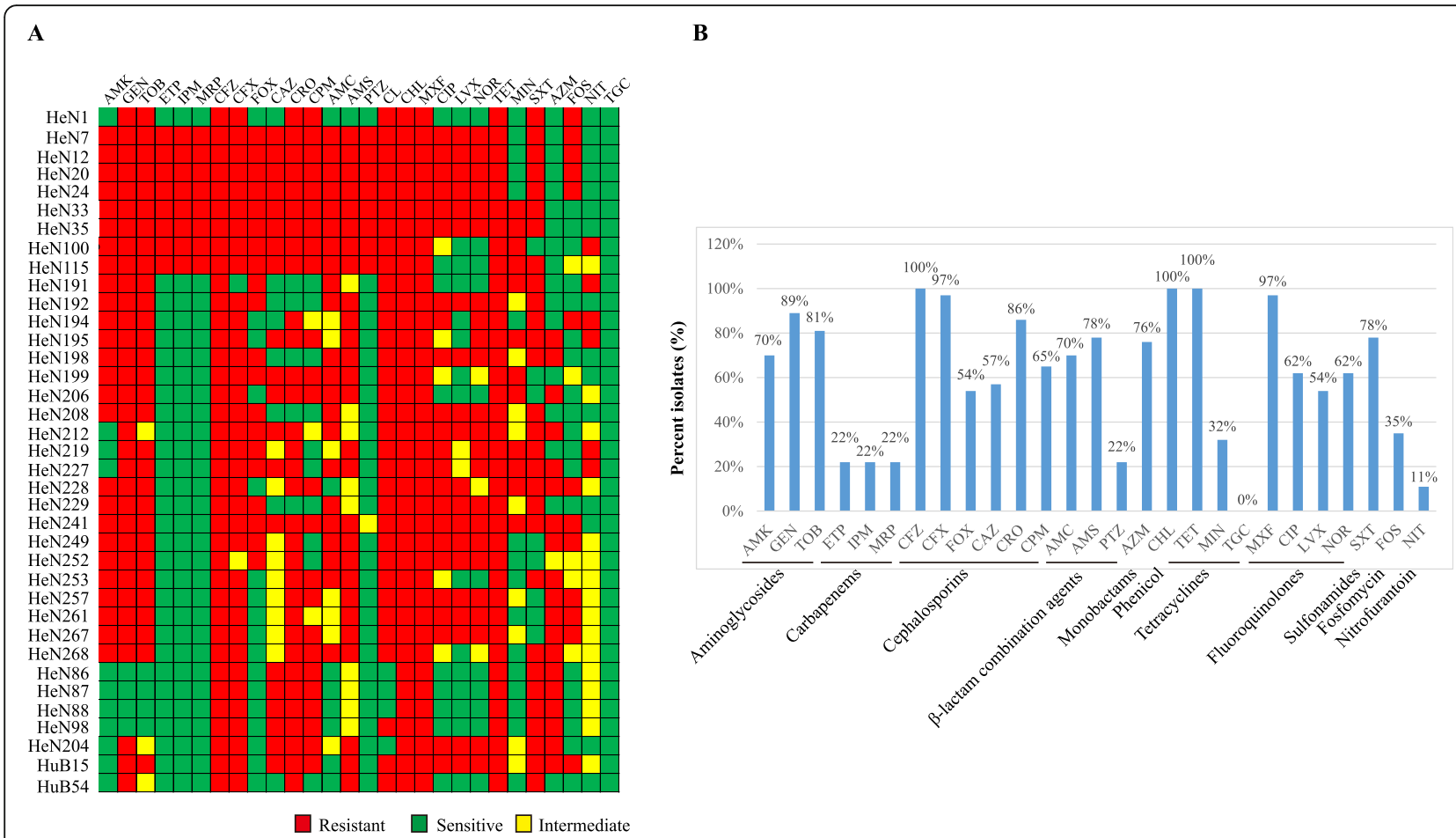

Fig. 2 Antimicrobial resistance phenotypes of $m c r-p o s i t i v e ~ E$. coli isolates. A Heat map showing antimicrobial resistance patterns of mcr-positive isolates in response to 28 test antibiotics. Small boxes marked in red, green and yellow represent resistant, sensitive and intermediate phenotypes. Results were interpreted according to the CLSI breakpoints (CLSI M100, 28th Edition). If a CLSI breakpoint was not available, an EUCAST breakpoint was used. B Column chart showing the percentages of isolates displaying phenotypes resistant to different tested antibiotics. AMK: amikacin, GEN: gentamicin, TOB: tobramycin, ETP: ertapenem, IPM: imipenem, MRP: meropenem, CFZ: cefazolin, CFX: cefuroxime, FOX: cefoxitin, CAZ: ceftazidime, CRO: ceftriaxone, CPM: cefepime, AMC: amoxicillin/ clavulanate, AMS: ampicillin/sulbactam, PTZ: piperacillin/tazobactam, CL: colistin, AZM: aztreonam, CHL: chloramphenicol, TET: tetracycline, MIN: minocycline, TGC: tigecycline, MXF: moxifloxacin, CIP: ciprofloxacin, LVX: levofloxacin, NOR: norfloxacin, SXT: trimethoprim/sulfamethoxazole, FOS: fosfomycin, NIT: nitrofurantoin

Antimicrobial susceptibility testing (AST) revealed that MIC of colistin in E. coli strains harboring $\mathrm{pMD}_{19}-\mathrm{mcr}$ $1^{\mathrm{R}}$, $\mathrm{pMD}_{19}-m c r-1^{\mathrm{S}}$ and $\mathrm{pMD}_{19}-\mathrm{T}$ were $2 \mu \mathrm{g} / \mathrm{mL}, 0.5 \mu \mathrm{g} /$ $\mathrm{mL}$ and $0.25 \mu \mathrm{g} / \mathrm{mL}$, respectively.

\section{Antimicrobial resistance phenotypes of colistin-resistant E. coli and mcr-positive E. coli}

AST revealed that the 37 mcr-positive isolates showed severe resistance profiles. All of them were resistant to more than nine types of tested antibiotics; over $80 \%$ $(81.08 \%, 30 / 37)$ of the isolates were resistant to more than 15 types of tested antibiotics; and approximately $24 \%(24.32 \%, 9 / 37)$ isolates were resistant to 20 types of antibiotics (Fig. 2A). Among antibiotics tested here, resistance to cefazolin (CFZ), cefuroxime (CFX), chloramphenicol (CHL), moxifloxacin (MXF) and tetracycline (TET) were the common phenotypes, and all $\mathrm{mcr}$-positive isolates were resistant to these five types (Fig. 2B). In particular, approximately $22 \%$ mcr-positive isolates were resistant to three carbapenem antibiotics tested here: imipenem (IPM), meropenem (MRP) and ertapenem (ETP) (Fig. 2B).

\section{Detection of antimicrobial resistance genes}

detection of ARGs showed that over 70\% mcr-positive isolates were positive for $r m t D(94.59 \%, 35 / 37)$, floR (94.59\%, 35/37), bla TEM $(91.89 \%, 34 / 37)$, tetA $(89.19 \%$, $33 / 37)$, sul2 $(75.68 \%, 28 / 37)$, sul3 $(75.68 \%, 28 / 37)$ and $r m t B(70.27 \%, 26 / 37)$. However, fewer than $50 \%$ isolates were positive for sul1 (48.65\%, 18/37), bla $a_{\mathrm{NDM}}(29.73 \%$, 11/37), armA $(16.22 \%, 6 / 37)$, tetB $(10.81 \%, 4 / 37)$, aac (6') $-\mathrm{Ib}(8.11 \%, 3 / 37)$, and $\operatorname{tet} M(5.41 \%, 2 / 37)$. None of the isolates were positive for $r m t A, r m t C$, qnrA, qnrB, $q n r C, q n r D$, tetC, bla $a_{\mathrm{SHV}}$ or bla $a_{\mathrm{CTX}-\mathrm{M}}$ (Fig. 3A).

Among the detected ARGs, all $\mathrm{mcr}$-positive isolates were positive for ARGs accounting for resistance to sulfonamides (sul1, sul2 and/or sul3), while $97 \%$ isolates were positive for ARGs accounting for resistance to aminoglycosides $(r m t D, r m t B$ and/or armA). Approximately 95\% isolates were positive for ARGs for resistance to tetracyclines (tet $A$, tet $B$ and/or tet $M$ ) and for resistance to phenicol $(f l o R)$. Totally $92 \%$ isolates were positive for ARGs accounting for resistance to $\beta$-lactams in addition to carbapenems $\left(b l a_{\text {TEM }}\right)$, and $32 \%$ isolates were positive for ARGs for resistance to carbapenems $\left(b l a_{\mathrm{NDM}}\right)$. Only 


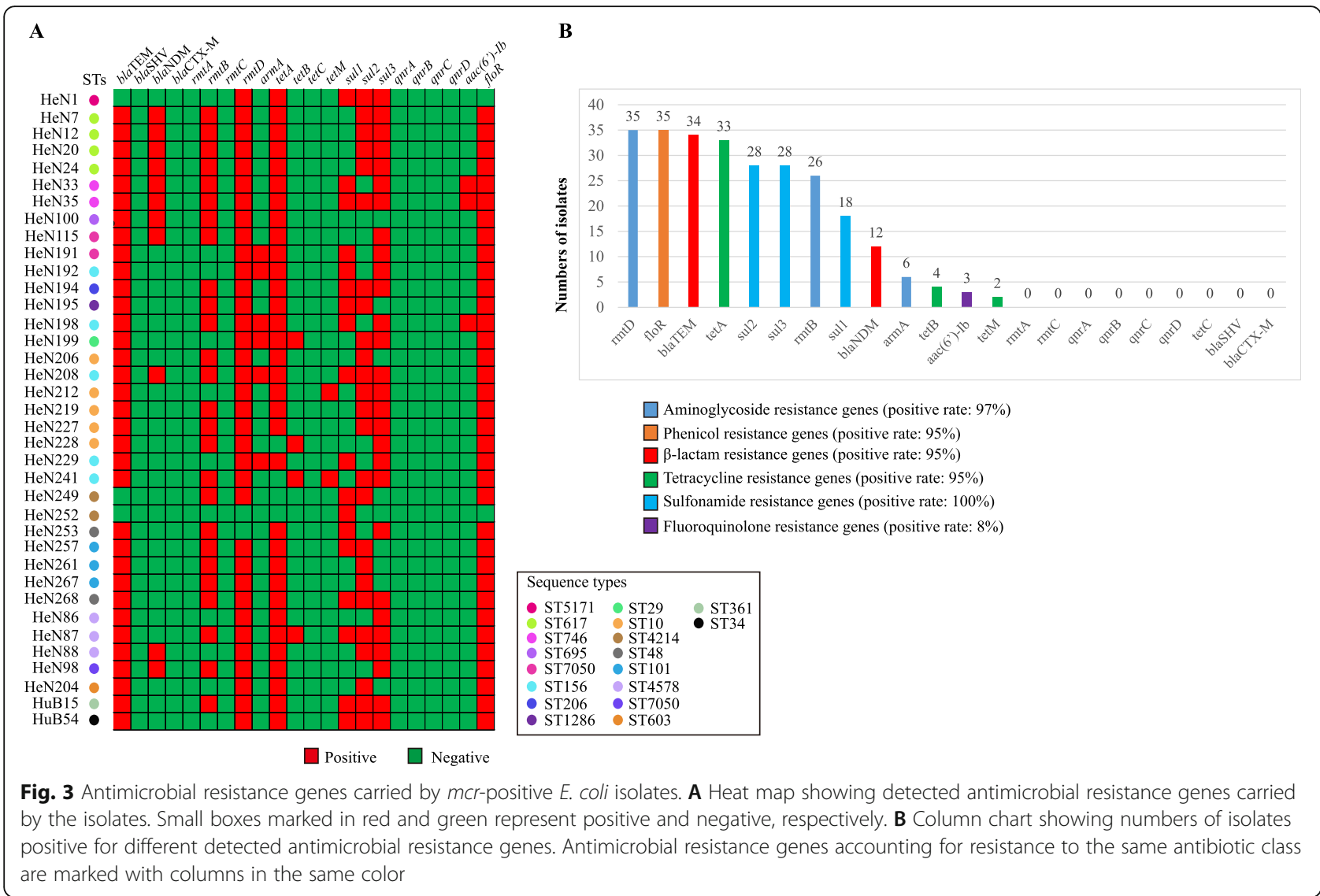

$8 \%$ isolates were positive for fluoroquinolone-resistant ARGs $\left(a a c\left(6^{\prime}\right)-I b\right)$ (Fig. 3B).

\section{Sequence types of colistin-resistant $E$. coli and $m c r-$ positive E. coli}

MLST (multilocus sequence typing) analysis identified 17 types of sequence for $37 \mathrm{mcr}$-positive isolates, including ST10 (5/17), ST156 (5/17), ST617 (4/17), ST101 (3/ 17), ST7050 (3/17), ST4578 (3/17), ST48 (2/17), ST746 (2/17), ST4214 (2/17), ST34 (1/17), ST603 (1/17), ST29 (1/17), ST1286 (1/17), ST206 (1/17), ST695 (1/17), ST5171 (1/17), and ST361 (1/17) (Fig 4). Phylogenetic trees constructed based on MLST data revealed that several sequence types showed close relationships (Fig. 4).

\section{Conjugation of mcr-carrying plasmids}

To assess the transferability of $m c r$-bearing plasmids, bacterial conjugation experiments were performed between $m c r$-carrying E. coli and recipient E. coli C600. Results showed that $m c r$-bearing plasmids from 25 isolates in this study were conjugated, and the conjugation frequencies ranged from $1.7 \times 10^{-6}$ to $4.1 \times 10^{-3}$ per recipient (Table 2). AST results revealed that conjugating mcr-bearing plasmids conferred a colistin resistance phenotype to the recipient $E$. coli. MIC of colistin for strain C600 was lower than $1 \mu \mathrm{g} / \mathrm{mL}$ while it increased to $4 \mu \mathrm{g} / \mathrm{mL}$ for transconjugants (Table 3 ).

\section{Types of $m c r$-carrying plasmids}

PCR detection of above 25 transconjugants revealed there were six plasmid types, namely IncX4 (14/25), FrepB (4/25), IncI2 (3/25), IncHI2 (2/25), FIB (1/25) and IncI1 $(1 / 25)$. Among these plasmid types, IncX4 was the most frequently detected, being found in 14 of the 25 transconjugants.

\section{Discussion}

The rapid increase and dissemination of colistinresistant Enterobacteriaceae as well as other gramnegative bacteria carrying plasmid-borne $\mathrm{mcr}$ genes in both humans and animals pose a major threat to global public health (Paterson and Harris 2016). Therefore, an extensive number of studies have monitored the prevalence and isolation of bacteria carrying plasmid-borne $m c r$ genes in both medical and veterinary environments in recent years (Ilbeigi et al. 2021; Snyman et al. 2021; Tufic-Garutti et al. 2021). In this study, we performed an isolation and microbiological characterization of colistin-resistant and $m c r$-positive $E$. coli isolates from three provinces in Central China. Our study revealed 


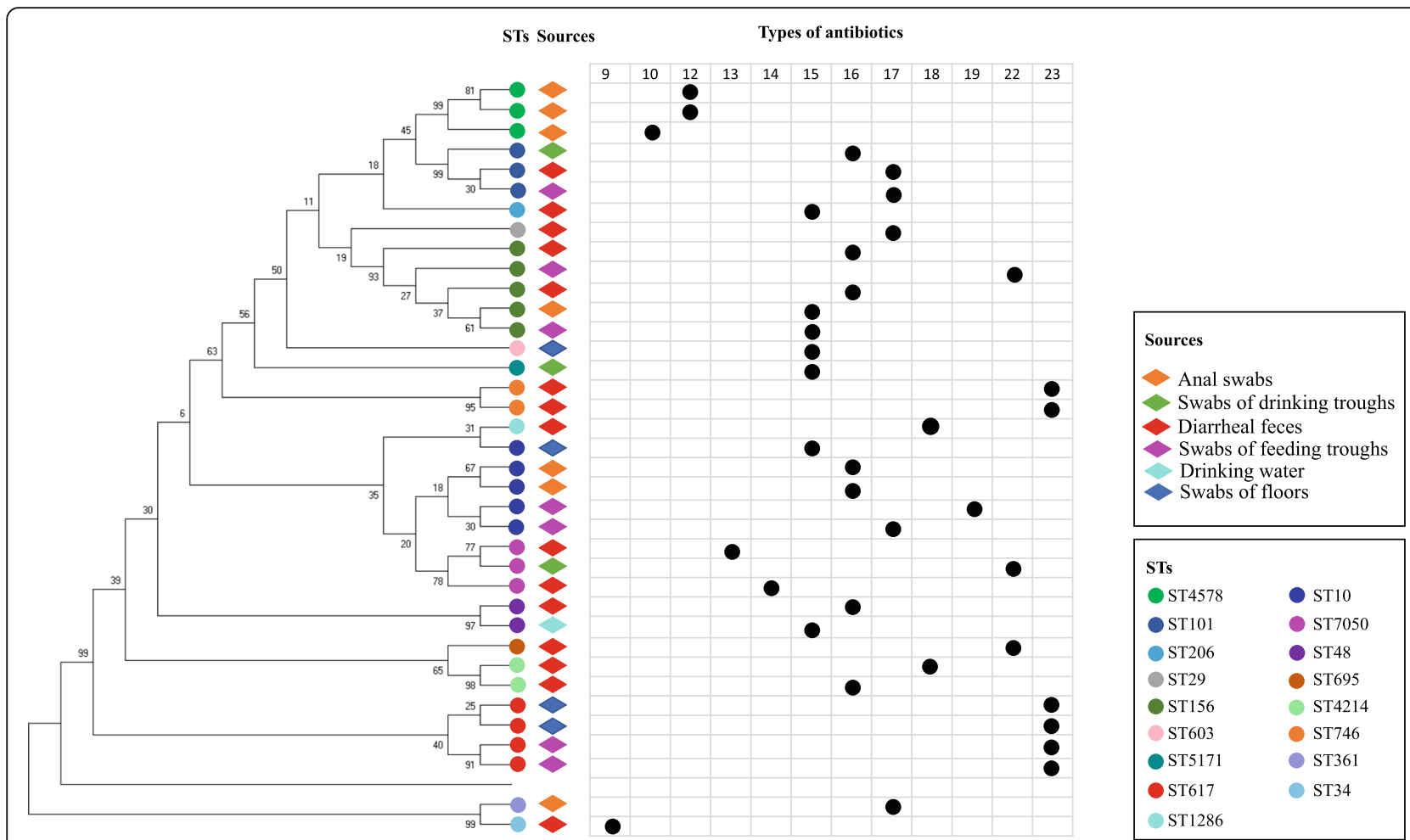

Fig. 4 Sequence types of mcr-positive E. coli isolates. The left panel shows a phylogenetic tree constructed based on the multilocus sequence typing (MLST) data, and the right panel shows the numbers of antibiotics that each isolate resists

total isolation rates of $5.56 \%(33 / 594)$ for colistinresistant E. coli and $6.23 \%$ (37/594) for $m c r$-positive $E$. coli. Both rates were much lower than those reported previously in China. In a recent study, frequency of colistin resistance in E. coli from pigs was $24.1 \%$ in 12 provinces of China from 2013-2014 (Huang et al. 2017), while in another study, prevalence of colistin-resistant $m c r-1$-harboring $E$. coli isolates in pigs from 14 Chinese provinces was as high as 45\% in 2016 (Shen et al. 2020). The relatively low prevalence rate determined in this study may be due to China's policy of banning the use of colistin as a growth promoter in livestock in China (Wang et al. 2020b). Following its implementation in 2017, it showed significant effects on reducing colistin resistance in both animals and humans in China. For example, a recent study showed that prevalence of colistin-

Table 2 Conjugation frequencies of mcr-carrying plasmids

\begin{tabular}{llcc}
\hline Conjugants & Frequencies (per recipient) & Conjugants & Frequencies (per recipient) \\
\hline HeN1C & $2.1 \times 10^{-5}$ & HeN208C & $1.6 \times 10^{-5}$ \\
HeN7C & $1.1 \times 10^{-5}$ & HeN219C & $1.3 \times 10^{-4}$ \\
HeN20C & $4.8 \times 10^{-5}$ & HeN227C & $6.9 \times 10^{-4}$ \\
HeN24C & $2.2 \times 10^{-5}$ & HeN228C & $7.6 \times 10^{-4}$ \\
HeN33C & $3.9 \times 10^{-3}$ & HeN241C & $3.8 \times 10^{-5}$ \\
HeN35C & $1.6 \times 10^{-3}$ & HeN249C & $2.3 \times 10^{-3}$ \\
HeN115C & $2.6 \times 10^{-3}$ & HeN252C & $4.1 \times 10^{-3}$ \\
HeN191C & $1.9 \times 10^{-4}$ & HeN253C & $8.1 \times 10^{-4}$ \\
HeN194C & $1.2 \times 10^{-5}$ & HeN12C & $2.0 \times 10^{-5}$ \\
HeN195C & $1.0 \times 10^{-5}$ & HeN268C & $1.6 \times 10^{-4}$ \\
HeN198C & $1.7 \times 10^{-6}$ & HeN98C & $3.4 \times 10^{-4}$ \\
HeN199C & $3.9 \times 10^{-5}$ & HuB15C & $4.2 \times 10^{-4}$ \\
HeN206C & $3.6 \times 10^{-4}$ & & \\
\hline
\end{tabular}


Table 3 Minimum inhibitory concentration (MIC) analysis of colistin for transconjugants and the recipient E. coli C600

\begin{tabular}{lclc}
\hline Conjugants & MIC $(\boldsymbol{\mu g} / \mathbf{m L})$ & Conjugants & MIC $(\boldsymbol{\mu g} / \mathbf{m L})$ \\
\hline HeN1C & 4 & HeN208C & 4 \\
HeN7C & 4 & HeN219C & 4 \\
HeN20C & 4 & HeN227C & 4 \\
HeN24C & 4 & HeN228C & 4 \\
HeN33C & 4 & HeN241C & 4 \\
HeN35C & 4 & HeN249C & 4 \\
HeN115C & 4 & HeN252C & 4 \\
HeN191C & 4 & HeN253C & 4 \\
HeN194C & 4 & HeN12C & 4 \\
HeN195C & 4 & HeN268C & 4 \\
HeN198C & 4 & HeN98C & 4 \\
HeN199C & 4 & HuB15C & 4 \\
HeN206C & 4 & C600 & $\leq 1$ \\
\hline
\end{tabular}

resistant $m c r-1$-harboring $E$. coli isolates in pigs from 14 Chinese provinces decreased from $45 \%$ (in 2016) to $19 \%$ after one year banning, with a remarkable reduction occurring in 10 of 14 surveyed provinces $(P<0.0001)$ (Shen et al. 2020). In the same study, a significant decrease in prevalence of $m c r-1$-harboring $E$. coli among farm pigs, from $76 \%$ in 2016 to $24 \%$ in 2018 ( $P<$ 0.0001), was also observed in Guangzhou, the capital of Guangdong Province in South China.

PCR detection results of this research revealed that all colistin-resistant $E$. coli were positive for $m c r-1$ (Fig. 1C, Table 1), suggesting that colistin resistance phenotype in these isolates was conferred by this gene (Liu et al. 2016). It's worth noting that MIC of colistin on colistinresistant $m c r$ - 1 -harboring $E$. coli isolates determined in this study were low $(4 \mu \mathrm{g} / \mathrm{ml}$ or $8 \mu \mathrm{g} / \mathrm{ml}$; Table 1$)$, which is consistent with those of other studies (Liu et al. 2016; Quan et al. 2017). This low value might have occurred because plasmid-mediated colistin resistance gene $\mathrm{mcr}-1$ generally confers low-level resistance (Poirel et al. 2017; $\mathrm{Zhu}$ et al. 2021). However, four mcr-1-positive isolates that did not display colistin resistance phenotypes were also identified (Fig. 1C, Table 1). Notably, this type of $E$. coli has been reported recently, but the underlying mechanism remains to be elucidated (Li et al. 2018). By nucleotide sequencing and sequence alignment analyses, $m c r-1$ gene carried by colistin-sensitive isolates showed an insertion of several bases, which caused an insertion of three amino acid residues ("LDT") at sites 314 316 in the protein (Fig. 1D). These insertions were located in the catalytic domain of MCR-1 (residues 215-541) (Stojanoski et al. 2016), which may thereby lead to the disruption of catalytic activity of this protein. Next, we intend to analyze structure of these inactive MCR proteins to reveal the non-working mechanisms. However, this hypothesis might be partly supported by our AST assays in different TOP10 E. coli transformants containing various plasmids (pMD19- $m c r-1^{\mathrm{R}}$, pMD19$m c r-1^{\mathrm{S}}$ and $\mathrm{pMD} 19-\mathrm{T}$ ), which revealed that MIC of transformants containing pMD19- $m c r-1^{\mathrm{S}}$ was $0.5 \mu \mathrm{g} /$ $\mathrm{mL}$, while that of transformants containing pMD19-mor$1^{\mathrm{R}}$ was $2 \mu \mathrm{g} / \mathrm{mL}$ (interpreted as colistin-resistant according to the EUCAST breakpoint).

This work also revealed that all $m c r-1$-positive E. coli isolates from pig farms had severe antimicrobial resistance profiles. They were also resistant to antibiotics commonly used in clinic, including World Health Organization (WHO)-listed important antibiotics such as aminoglycosides, broad-spectrum cephalosporins and other $\beta$-lactams, and fluoroquinolones (Fig. 2). All of these isolates could be defined as multidrug-resistant bacteria and even extensively resistant bacteria, according to the international expert proposal for interim standard definitions for acquired resistance (Magiorakos et al. 2012). Similar findings have also been reported in other parts of China (Cheng et al. 2020; Tong et al. 2018; Yuan et al. 2021), as well as other countries around the world (Clemente et al. 2019; Oh et al. 2020; Zając et al. 2019). The presence of these multidrugresistant $E$. coli strains at farm level poses a high probability of spreading them to humans along pork supply chain since E. coli is a common food contaminating bacterium as well as a foodborne pathogen (Batz et al. 2011). In particular, eight $m c r$-positive isolates were also resistant to three tested carbapenems (IPM, MRP, ETP) (Fig. 2).

Previously, we reported the genomic characteristics of these colistin and carbapenem co-resistant isolates, and showed that these worrisome phenotypes were conferred by plasmid-borne ARGs $\left(m c r-1\right.$ and $\left.b l a_{\mathrm{NDM}-1}\right)$ with conjugation capacity (Peng et al. 2019a, b). It should be remembered that both colistin and carbapenems are recognized as last-resort antibiotics for treating infections caused by multidrug-resistant gram-negative pathogens ( $\mathrm{Du}$ et al. 2016). The existence of these isolates in food animals may lead to having no antibiotics available in clinical settings if they are transmitted to humans. The continuous monitoring of these colistin and carbapenem co-resistant isolates in both medical and veterinary environments is necessary and important. Corresponding to the serious resistance phenotypes determined here, ARG detection also revealed a serious condition in which resistance genes were found among these $\mathrm{mcr}$-positive isolates. More than $90 \%$ isolates were positive for ARGs accounting for resistance to sulfonamides (sul1, sul2 and/or sul3), aminoglycosides ( $r m t D, r m t B$ and/or armA), tetracyclines (tet $A$, tetB and/ or tetM), phenicol (floR) and $\beta$-lactams $\left(\right.$ bla $\left._{\mathrm{TEM}}\right)$ (Fig. 3). Since these ARGs are frequently disseminated through 
horizontal transfer, with the help of plasmids and other mobile genetic elements, these $\mathrm{mcr}$-positive isolates harboring multiple ARGs may represent a major reservoir of resistance genes that may be responsible for treatment failures in both human and veterinary medicine (Poirel et al. 2018).

By performing MLST analysis, a heterogeneous group of sequence types were determined for $37 \mathrm{mcr}$-positive isolates. ST10 (5/37), ST156 (5/37) and ST617 (4/37) were the most predominant (Fig. 4). It has been reported that $E$. coli ST10 and its related types were frequently recovered from livestock, food and human intestinal samples. A higher prevalence of plasmid-borne ARGs was found in these types compared to others (Manges et al. 2017; Matamoros et al. 2017; Oteo et al. 2009). Similar to these reports, E. coli ST10 and its related types (ST1286, ST7050) showed severe AMR profiles and possessed many ARGs in addition to $m c r-1$, including ESBL-encoding gene bla $a_{\text {TEM }}$ (Figs. 2 and 3), suggesting that these sequence types pose a threat to public health. In addition, $11 \mathrm{NDM}$ and MCR co-producing E. coli isolates were determined in this study (Fig. 3), and they were assigned to ST617 (4/11), ST746 (2/11), ST7050 (2/11), ST156 (1/11), ST695 (1/11) and ST4578 (1/11) (Fig. 4). Although most of these sequence types represent novel $E$. coli co-producing MCRs and NDMs, E. coli strains such as ST617 and ST746, have been recovered from both patients and diseased pigs (Gedebjerg et al. 2015; Hayer et al. 2020; Tian et al. 2020; Wu et al. 2018). Considering that $\mathrm{mcr}$ commonly confers resistance to the last-resort antibiotic colistin and $b l a_{\mathrm{NDM}}$ commonly confers resistance to another last-resort antibiotic class, carbapenems, for treating infections caused by gram-negative pathogens ( $\mathrm{Du}$ et al. 2016), the recovery of MCR and NDM co-produced in E. coli with potentially pathogenic sequence types should receive more attention. These bacteria might cause infections for which there are no effective antibiotics available in clinic. Continuous monitoring of these bacteria in both humans and animals is necessary.

mor genes are generally disseminated by plasmids (Liu et al. 2016; Yi et al. 2017). To understand whether $\mathrm{mcr}$ gene harbored in these $\mathrm{mcr}$-positive isolates is carried by plasmids and their plasmid types, plasmid conjugation experiments were first performed. Results revealed that $\mathrm{mcr}$ gene in $25 \mathrm{mcr}$-positive isolates was conjugated to recipient $E$. coli, with conjugation frequencies ranging from $1.7 \times 10^{-6}$ to $4.1 \times 10^{-3}$ per recipient (Table 2). More importantly, the conjugation of these $\mathrm{mcr}$ genes conferred a colistin resistance phenotype to the recipient bacterium (Table 3). These findings indicate that $\mathrm{mcr}$ genes carried by the isolates from pig farms in this study possess transferability and could mediate the transmission of colistin resistance. PCR typing analysis of plasmids harbored in the 25 transconjugants revealed six types of plasmid replicons, specifically, IncX4 (14/25), FrepB (4/25), IncI2 (3/25), IncHI2 (2/25), FIB (1/25) and IncI1 $(1 / 25)$. These findings are in part consistent with those from other reports, in which researchers also found that $m c r$ genes were mostly detected in $E$. coli harboring plasmids IncX4, IncHI2, IncI2 and/or IncI1 (Al-Mir et al. 2021; Höfle et al. 2020; Olowo-Okere and Yacouba 2020; Song et al. 2020; Zelendova et al. 2020). While less common, $m c r$ gene in E. coli carried by FIB and FrepB plasmids have also been documented (Shafiq et al. 2021).

\section{Conclusion}

In summary, we characterized drug resistance phenotypes, ARG profiles, sequence types, and putative plasmid types of colistin-resistant and/or $m c r$-positive $E$. coli isolates from pig farms in Central China in this study. Results revealed that several $m c r$-positive $E$. coli did not display a colistin resistance phenotype, which might be because base mutations were present, thereby leading to gene dysfunction. Notably, $m c r$-positive $E$. coli isolates determined in this study displayed severe AMR profiles, carried multiple ARGs, including those associated with great public health concerns, indicating that these E. coli isolates pose a threat to human health. This research also revealed a heterogeneous group of sequence types for $m c r$-positive $E$. coli isolates and several sequence types, such as ST617 and ST746, which reportedly correlated with diseases in both humans and pigs. These clones should receive more attention. In addition, we found that $m c r$ genes in E. coli isolates from pig farms in Central China were most likely to carry several types of plasmids, and most of these plasmids possessed transferability and could help disseminate colistin resistance. Prevalence of colistin-resistant and/or $\mathrm{mcr}$-positive $E$. coli in pig farms of China will be continuously monitored in the future.

\section{Materials and methods}

\section{Sample collection and bacterial isolation}

The study design is shown in Fig. 1A. Between 2018 and 2019, a total of 594 samples were collected, including fecal samples from diarrheal pigs, anal swabs from healthy pigs, and swabs of feeding and drinking troughs and floors from nine farms in Henan, Hubei and Hunan provinces in Central China for E. coli isolation (Fig. 1B). Samples were maintained in sterilized buffered peptone water (BPW) and shipped on ice to laboratory for immediate treatment. To improve isolation efficacy, each collected samples was pre-incubated in Luria-Bertani (LB) broth (Sigma-Aldrich, MO, USA) at $37^{\circ} \mathrm{C}$ for $12 \mathrm{~h}$. Afterwards, sample culture was streaked on MacConkey agars and incubated at $37^{\circ} \mathrm{C}$ for $12 \mathrm{~h}$. Presumptive 
colonies with similar morphological characteristics to those of $E$. coli were selected for further confirmation by 16S rRNA gene sequencing and PCR amplification of seven housekeeping genes ( $a d k$, fumC, gyrA, icd, $m d h$, purA and recA) in E. coli, as described previously (Wirth et al. 2006). On each agar plates, five colonies were selected, but only one confirmed E. coli colony was included for further study.

\section{Screening colistin-resistant isolates and $m c r$-positive isolates}

To screen isolates with a colistin resistance phenotype, recovered E. coli isolates were streaked onto Müller-Hinton $(\mathrm{MH})$ agar containing $2 \mu \mathrm{g} / \mathrm{mL}$ colistin (concentration chosen based on the European Committee on Antimicrobial Susceptibility Testing [EUCAST] clinical breakpoint 2018) and cultured at $37^{\circ} \mathrm{C}$ for 12 h. E. coli ATCC 25922 was used as a quality control. In parallel, all $E$. coli isolates were also selected as $m c r$-positive strains by using PCR with primers listed in Table S1. PCR assays were performed in a $20 \mu \mathrm{L}$ mixture containing $1 \mu \mathrm{L}$ bacterial DNA template, $1 \mu \mathrm{L}$ forward or reverse primers, 10 $\mu \mathrm{L} 2 \times$ Master Mix (Vazyme, Nanjing, China), and 7 $\mu \mathrm{L} \mathrm{dd}_{2} \mathrm{O}$. Thermocycling conditions were $95^{\circ} \mathrm{C}$ for 5 min, followed by 30 cycles of $95^{\circ} \mathrm{C}$ for $30 \mathrm{~s}$, annealing at $51 \sim 60^{\circ} \mathrm{C}$ for $30 \mathrm{~s}$ (Table S1), and $72^{\circ} \mathrm{C}$ for $2 \mathrm{~min}$ $30 \mathrm{~s}$, with a final extension at $72^{\circ} \mathrm{C}$ for $5 \mathrm{~min}$. PCR products were analyzed by electrophoresis on a $1 \%$ agarose gel.

\section{Antimicrobial susceptibility testing}

The resistance phenotypes of colistin-resistant isolates and $m c r$-positive isolates were determined using broth microdilution method in accordance with the protocol published by the United States Clinical \& Laboratory Standard Institute (CLSI document M100, 28th Edition). A total of 28 types of antibiotics, namely amikacin (AMK), gentamicin (GEN), tobramycin (TOB), imipenem (IPM), meropenem (MRP), ertapenem (ETP), colistin (CL), cefazolin (CFZ), cefuroxime (CFX), cefoxitin (FOX), ceftazidime (CAZ), ceftriaxone (CRO), cefepime (CPM), chloramphenicol (CHL), fosfomycin (FOS), nitrofurantoin (NIT), ciprofloxacin (CIP), levofloxacin (LVX), moxifloxacin (MXF), norfloxacin (NOR), minocycline (MIN), tetracycline (TET), aztreonam (AZM), tigecycline (TGC), amoxicillin/clavulanate (AMC), ampicillin/sulbactam (AMS), piperacillin/tazobactam (PTZ) and trimethoprim/sulfamethoxazole (SXT) were assessed. Results were interpreted using the CLSI breakpoints (CLSI M100, 28th Edition). If a CLSI breakpoint was not available, a EUCAST breakpoint was used. E. coli ATCC 25922 was used for quality control.

\section{Detection of antimicrobial resistance genes}

Antimicrobial resistance genes (ARGs) harbored in screened colistin-resistant $E$. coli were detected by PCR with primers listed in Table S1. A total of 22 types of ARGs that conferred resistance to six antibiotic classes were detected. PCR assays were performed the same as isolate screening described above.

\section{Multilocus sequence typing}

Multilocus sequence typing (MLST) was performed by following protocol published in the E. coli MLST database (https://enterobase.warwick.ac.uk/species/ecoli/). Sequence types were assigned based on the alleles of seven housingkeeping genes in E. coli ( $a d k$, fumC, gyrA, icd, $m d h$, purA and $\operatorname{rec} A$ ). Primers used for amplifying these genes were listed in Table S1. PCR assays were performed in a $30 \mu \mathrm{L}$ mixture containing $1 \mu \mathrm{L}$ bacterial DNA template, $1 \mu \mathrm{L}$ forward or reverse primers, $15 \mu \mathrm{L} 2 \times$ Phanta Master Mix (Vazyme, Nanjing, China), and $12 \mu \mathrm{L} d \mathrm{ddH}_{2} \mathrm{O}$. PCR assays were performed the same as isolate screening described above. The confirmed products were sent for nucleotide sequencing and DNA sequences were submitted to the $E$. coli MLST database (https:/enterobase.warwick.ac.uk/species/ ecoli/) for sequence type determination.

\section{Plasmid conjugation}

Plasmid conjugation assays between mor-positive E. coli (donor) and rifampin-resistant E. coli C600 (recipient) were performed on a nitrocellulose membrane, as described previously (Peng et al. 2019a). In brief, a mid-log phase donor and the recipient strains $\left(\mathrm{OD}_{600}=0.5 \sim 0.6\right)$ were mixed at a ratio of 1:3 $(v / v)$. The bacterial mixture was then spotted onto a nitrocellulose membrane that was pre-plated on LB agar. After a $12 \mathrm{~h}$ incubation at $37^{\circ} \mathrm{C}$, bacteria on the membrane were washed off using $\mathrm{LB}$ broth and were shaken at $37^{\circ} \mathrm{C}$ for $4 \mathrm{~h}$. Lastly, the transconjugants were selected on LB agar plates laced with rifampin $(1000 \mu \mathrm{g} / \mathrm{mL}$; our pretests showed that all the $E$. coli donor strains could be inhibited by this concentration) plus colistin $(2 \mu \mathrm{g} / \mathrm{mL})$. MIC for colistin of transconjugants were determined using broth microdilution method as mentioned above. E. coli ATCC 25922 was used as quality control.

\section{Plasmid typing}

Putative types of $\mathrm{mcr}$-carrying plasmids were determined by PCR assays with primers listed in Table S1. PCR assays were performed the same as isolate screening described above.

\section{Abbreviations}

AMK: Amikacin; GEN: Gentamicin; TOB: Tobramycin; ETP: Ertapenem; IPM: Imipenem; MRP: Meropenem; CFZ: Cefazolin; CFX: Cefuroxime; FOX: Cefoxitin; CAZ: Ceftazidime; CRO: Ceftriaxone; CPM: Cefepime; AMC: Amoxicillin/clavulanate; AMS: Ampicillin/sulbactam; PTZ: Piperacillin/ 
tazobactam; CL: Colistin; AZM: Aztreonam; CHL: Chloramphenicol; TET: Tetracycline; MIN: Minocycline; TGC: Tigecycline; MXF: Moxifloxacin; CIP: Ciprofloxacin; LVX: Levofloxacin; NOR: Norfloxacin; SXT: Trimethoprim/ sulfamethoxazole; FOS: Fosfomycin; NIT: Nitrofurantoin

\section{Supplementary Information}

The online version contains supplementary material available at https://doi. org/10.1186/s44149-021-00009-5.

Additional file 1: Table S1. Primers used in the present study.

\section{Authors' contributions}

Z.P., H.C., and X.W. contributed to the conception and design of this work: Z.P., X.Z., X.L., Z.H., Z.L., and C.J. participated in sample collection, laboratory experiments and data analysis; Z.P., and X.W. drafted the manuscript; and Z.P., M.D., C.T., H.C., and X.W. revised the manuscript. All the authors read and approved the final manuscript.

\section{Funding}

This work was supported in part by the National Key R\&D Program of China (grant numbers: 2017YFC1600103 and 2017YFC1600101), the Natural Science Foundation of Hubei Province (grant number: 2020CFB525), the China Agriculture Research System of MOF and MARA (grant number CARS-35), and the Walmart Foundation (Project \# 61626817). Zhong Peng acknowledges the financial support from the China Postdoctoral Science Foundation (grant number: 2018 M640719). The funder had no role in the study design, data collection, data analysis, data interpretation, or writing of the manuscript.

\section{Availability of data and materials}

Not applicable.

\section{Declarations}

Ethics approval and consent to participate

Not applicable.

\section{Consent for publication}

Not applicable.

\section{Competing interests}

There is no conflict of interest to declare.

\begin{abstract}
Author details
${ }^{1}$ State Key Laboratory of Agricultural Microbiology, College of Veterinary Medicine, Huazhong Agricultural University, No.1 Shizishan Street, Hongshan District, Wuhan, China. ${ }^{2}$ Key Laboratory of Preventive Veterinary Medicine in Hubei Province, The Cooperative Innovation Center for Sustainable Pig Production, Huazhong Agricultural University, Wuhan, China. ${ }^{3}$ Key Laboratory of Development of Veterinary Diagnostic Products, Ministry of Agriculture and Rural Affairs, Huazhong Agricultural University, Wuhan, China. ${ }^{4}$ International Research Center for Animal Disease, Ministry of Science and Technology, Huazhong Agricultural University, Wuhan, China.
\end{abstract}

\section{Received: 15 April 2021 Accepted: 25 May 2021}

Published online: 12 July 2021

\section{References}

Al-Mir, H., M. Osman, A. Drapeau, M. Hamze, J.Y. Madec, and M. Haenni. 2021. WGS analysis of clonal and plasmidic epidemiology of colistin-resistance mediated by mcr genes in the poultry sector in Lebanon. Frontiers in Microbiology 12: 624194. https://doi.org/10.3389/fmicb.2021.624194.

Andrade, F.F., D. Silva, A. Rodrigues, and C. Pina-Vaz. 2020. Colistin update on its mechanism of action and resistance, present and future challenges. Microorganisms 8 (11): 1716. https://doi.org/10.3390/microorganisms 8111716.

Batz, M.B., S. Hoffmann, and J.G. Morris. 2011. Ranking the risks: The 10 pathogenfood combinations with the greatest burden on public health. Gainesville: Emerging Pathogens Institute., University of Florida http://www.rwjf.org/files/ research/72267report.pdf.
Cheng, P., Y.Q. Yang, J.C. Zhang, F.L. Li, X.T. Li, H.B. Liu, M. Ishfaq, G.F. Xu, and X.Y. Zhang. 2020. Antimicrobial resistance and virulence profiles of mcr-1-positive Escherichia coli isolated from swine farms in Heilongjiang Province of China. Journal of Food Protection 83 (12): 2209-2215. https://doi.org/10.4315/JFP-20-190.

Clemente, L., V. Manageiro, I. Correia, A. Amaro, T. Albuquerque, P. Themudo, E. Ferreira, and M. Caniça. 2019. Revealing mcr-1-positive ESBL-producing Escherichia coli strains among Enterobacteriaceae from food-producing animals (bovine, swine and poultry) and meat (bovine and swine), Portugal, 2010-2015. International Journal of Food Microbiology 296: 37-42. https://doi. org/10.1016/j.jijfoodmicro.2019.02.006.

Du, H., L. Chen, Y.W. Tang, and B.N. Kreiswirth. 2016. Emergence of the mcr-1 colistin resistance gene in carbapenem-resistant Enterobacteriaceae. The Lancet Infectious Diseases 16 (3): 287-288. https://doi.org/10.1016/S1473-3 099(16)00056-6.

El-Sayed Ahmed, M.A.E.G., L.L. Zhong, C. Shen, Y.Q. Yang, Y. Doi, and G.B. Tian. 2020. Colistin and its role in the Era of antibiotic resistance: an extended review (2000-2019). Emerging Microbes \& Infections 9 (1): 868-885. https://doi. org/10.1080/22221751.2020.1754133.

Gedebjerg, A., H. Hasman, C.M. Sørensen, and M. Wang. 2015. An OXA-48producing Escherichia coli isolated from a Danish patient with no hospitalization abroad. Infectious Diseases (London, England) 47 (8): 593-595. https://doi.org/10.3109/23744235.2015.1019920.

Hayer, S.S., S. Lim, S. Hong, E. Elnekave, T. Johnson, A. Rovira, F. Vannucci, J.B. Clayton, A. Perez, and J. Alvarez. 2020. Genetic determinants of resistance to extended-spectrum cephalosporin and fluoroquinolone in Escherichia coli isolated from diseased pigs in the United States. mSphere 5 (5): e00990e00920. https://doi.org/10.1128/msphere.00990-20.

Höfle, U., J. Jose Gonzalez-Lopez, M.C. Camacho, M. Solà-Ginés, A. MorenoMingorance, J. Manuel Hernández, J. de la Puente, J. Pineda-Pampliega, J.I. Aguirre, F. Torres-Medina, et al. 2020. Foraging at solid urban waste disposal sites as risk factor for cephalosporin and colistin resistant Escherichia coli carriage in white storks (Ciconia ciconia). Frontiers in Microbiology 11: 1397. https://doi.org/10.3389/fmicb.2020.01397.

Huang, X.H., L.F. Yu, X.J. Chen, C.P. Zhi, X. Yao, Y.Y. Liu, S.J. Wu, Z.W. Guo, L.X. Yi, Z L. Zeng, et al. 2017. High prevalence of colistin resistance and $\mathrm{mcr}^{-1}$ gene in Escherichia coli isolated from food animals in China. Frontiers in Microbiology 8: 562. https://doi.org/10.3389/fmicb.2017.00562.

Ilbeigi, K., M. Askari Badouei, H. Vaezi, H. Zaheri, S. Aghasharif, and K. Kafshdouzan. 2021. Molecular survey of mcr1 and mcr2 plasmid mediated colistin resistance genes in Escherichia coli isolates of animal origin in Iran. BMC Research Notes 14 (1): 1-5. https://doi.org/10.1186/s13104-021-05519-6.

Li, B.Y., B.X. Ke, X. Zhao, Y.X. Guo, W.Q. Wang, X.X. Wang, and H.H. Zhu. 2018. Antimicrobial resistance profile of $\mathrm{mcr}-1$ positive clinical isolates of Escherichia coli in China from 2013 to 2016. Frontiers in Microbiology 9: 2514. https://doi. org/10.3389/fmicb.2018.02514

Li, J., R.L. Nation, and KS. Kaye. 2019a. Polymyxin antibiotics: from laboratory bench to bedside. Cham: Springer International Publishing. https://doi.org/10.1007/978-3-030-16373-0_3.

Li, J., R.L. Nation, and K.S. Kaye. 2019b. Polymyxin antibiotics: from laboratory bench to bedside. Cham: Springer International Publishing. https://doi.org/10.1007/ 978-3-030-16373-0_2

Li, J., R.L. Nation, and K.S. Kaye. 2019c. Polymyxin antibiotics: from laboratory bench to bedside. Cham: Springer International Publishing. https://doi.org/10.1007/ 978-3-030-16373-0_4.

Liu, Y.Y., Y. Wang, T.R. Walsh, L.X. Yi, R. Zhang, J. Spencer, Y. Doi, G.B. Tian, B.L. Dong, X.H. Huang, et al. 2016. Emergence of plasmid-mediated colistin resistance mechanism MCR-1 in animals and human beings in China: a microbiological and molecular biological study. The Lancet Infectious Diseases 16 (2): 161-168. https://doi.org/10.1016/S1473-3099(15)00424-7.

Magiorakos, A.P., A. Srinivasan, R.B. Carey, Y. Carmeli, M.E. Falagas, C.G. Giske, S. Harbarth, J.F. Hindler, G. Kahlmeter, B. Olsson-Liljequist, et al. 2012. Multidrugresistant, extensively drug-resistant and pandrug-resistant bacteria: an international expert proposal for interim standard definitions for acquired resistance. Clinical Microbiology and Infection 18 (3): 268-281. https://doi.org/1 0.1111/j.1469-0691.2011.03570.x

Manges, A.R., K. Mende, C.K. Murray, B.D. Johnston, E.V. Sokurenko, V. Tchesnokova, and J.R. Johnson. 2017. Clonal distribution and associated characteristics of Escherichia coli clinical and surveillance isolates from a military medical center. Diagnostic Microbiology and Infectious Disease 87 (4): 382-385. https://doi.org/10.1016/j.diagmicrobio.2017.01.007.

Matamoros, S., J.M. van Hattem, M.S. Arcilla, N. Willemse, D.C. Melles, J. Penders, T. N. Vinh, N.T. Hoa, M.C.J. Bootsma, P.J. van Genderen, et al. 2017. Global 
phylogenetic analysis of Escherichia coli and plasmids carrying the mcr-1 gene indicates bacterial diversity but plasmid restriction. Scientific Reports 7: 15364. https://doi.org/10.1038/s41598-017-15539-7.

Oh, S.S., J. Song, J. Kim, and J. Shin. 2020. Increasing prevalence of multidrugresistant mcr-1-positive Escherichia coli isolates from fresh vegetables and healthy food animals in South Korea. International Journal of Infectious Diseases 92: 53-55. https://doi.org/10.1016/j.ijid.2019.12.025.

Olowo-Okere, A., and A. Yacouba. 2020. Molecular mechanisms of colistin resistance in Africa: a systematic review of literature. Germs 10 (4): 367-379. https://doi.org/10.18683/germs.2020.1229.

Oteo, J., K. Diestra, C. Juan, V. Bautista, A. Novais, M. Pérez-Vázquez, B. Moyá, E. Miró, T.M. Coque, A. Oliver, et al. 2009. Extended-spectrum beta-lactamaseproducing Escherichia coli in Spain belong to a large variety of multilocus sequence typing types, including ST10 complex/A, ST23 complex/A and ST131/B2. International Journal of Antimicrobial Agents 34 (2): 173-176. https://doi.org/10.1016/j.jjantimicag.2009.03.006.

Paterson, D.L., and P.N.A. Harris. 2016. Colistin resistance: a major breach in our last line of defence. The Lancet Infectious Diseases 16 (2): 132-133. https://doi. org/10.1016/S1473-3099(15)00463-6.

Peng, Hu, Li, Li, Jia, Zhang, Wu, Chen, and Wang. 2019a. Characteristics of a colistin-resistant Escherichia coli ST695 harboring the chromosomallyencoded mcr-1 gene. Microorganisms 7 (11): 558. https://doi.org/10.3390/ microorganisms7110558.

Peng, Li, Hu, Li, Lv, Lei, Wu, Chen, and Wang. 2019b. Characteristics of carbapenem-resistant and colistin-resistant Escherichia coli co-producing NDM-1 and MCR-1 from pig farms in China. Microorganisms 7 (11): 482. https://doi.org/10.3390/microorganisms7110482.

Poirel, L., A. Jayol, and P. Nordmann. 2017. Polymyxins: antibacterial activity, susceptibility testing, and resistance mechanisms encoded by plasmids or chromosomes. Clinical Microbiology Reviews 30 (2): 557-596. https://doi.org/1 0.1128/CMR.00064-16

Poirel, L., J.Y. Madec, A. Lupo, A.K. Schink, N. Kieffer, P. Nordmann, and S. Schwarz 2018. Antimicrobial resistance in Escherichia coli. Microbiology Spectrum 6 (4): 289-316. https://doi.org/10.1128/microbiolspec.arba-0026-2017.

Quan, J.J., X. Li, Y. Chen, Y. Jiang, Z.H. Zhou, H.C. Zhang, L. Sun, Z. Ruan, Y. Feng, M. Akova, et al. 2017. Prevalence of mcr-1 in Escherichia coli and Klebsiella pneumoniae recovered from bloodstream infections in China: a multicentre longitudinal study. The Lancet Infectious Diseases 17 (4): 400-410. https://doi. org/10.1016/S1473-3099(16)30528-X.

Shafiq, M., J.H. Huang, J.M. Shah, I. Ali, S.U. Rahman, and L.P. Wang. 2021. Characterization and resistant determinants linked to mobile elements of ESBL-producing and mcr-1-positive Escherichia coli recovered from the chicken origin. Microbial Pathogenesis 150: 104722. https://doi.org/10.1016/j. micpath.2020.104722.

Shen, C., L.L. Zhong, Y.Q. Yang, Y. Doi, D.L. Paterson, N. Stoesser, F.R. Ma, M.A.E.G. El-Sayed Ahmed, S.Y. Feng, S.Y. Huang, et al. 2020. Dynamics of mcr-1 prevalence and mcr-1-positive Escherichia coli after the cessation of colistin use as a feed additive for animals in China: a prospective cross-sectional and whole genome sequencing-based molecular epidemiological study. The Lancet Microbe 1 (1): e34-e43. https://doi.org/10.1016/S2666-5247(20)30005-7.

Snyman, Y., S. Reuter, A.C. Whitelaw, L. Stein, M.R.B. Maloba, and M. Newton-Foot. 2021. Characterization of mcr-4.3 in a colistin-resistant Acinetobacter nosocomialis clinical isolate from Cape Town, South Africa. Journal of Global Antimicrobial Resistance 25: 102-106. https://doi.org/10.1016/j.jgar.2021.03.002.

Song, H.J., D.C. Moon, A.F. Mechesso, H.Y. Kang, M.H. Kim, J.H. Choi, S.J. Kim, S.S. Yoon, and S.K. Lim. 2020. Resistance profiling and molecular characterization of extended-spectrum/plasmid-mediated AmpC $\beta$-lactamase-producing Escherichia coli isolated from healthy broiler chickens in south Korea. Microorganisms 8 (9): 1434. https://doi.org/10.3390/microorganisms8091434.

Stojanoski, V., B. Sankaran, B.V.V. Prasad, L. Poirel, P. Nordmann, and T. Palzkill. 2016. Structure of the catalytic domain of the colistin resistance enzyme MCR-1. BMC Biology 14 (1): 81. https://doi.org/10.1186/s12915-016-0303-0.

Sun, J., H.M. Zhang, Y.H. Liu, and Y.J. Feng. 2018. Towards understanding MCR-like colistin resistance. Trends in Microbiology 26 (9): 794-808. https://doi.org/10.1 016/j.tim.2018.02.006.

Tian, D.X., B.J. Wang, H. Zhang, F. Pan, C. Wang, Y.Y. Shi, and Y. Sun. 2020. Dissemination of the blaNDM-5 gene via IncX3-type plasmid among Enterobacteriaceae in children. mSphere 5 (1): e00699-e00619. https://doi. org/10.1128/msphere.00699-19.

Tong, H.X., J.X. Liu, X.H. Yao, H.Y. Jia, J.C. Wei, D.H. Shao, K. Liu, Y.F. Qiu, Z.Y. Ma, and B.B. Li. 2018. High carriage rate of $\mathrm{mcr}-1$ and antimicrobial resistance profiles of mcr-1-positive Escherichia coli isolates in swine faecal samples collected from eighteen provinces in China. Veterinary Microbiology 225: $53-$ 57. https://doi.org/10.1016/j.vetmic.2018.09.018.

Tufic-Garutti, S.D.S., J.V.A.R. Ramalho, L.G.D.A. Longo, G.C. de Oliveira, G.T. Rocha, L. C. Vilar, M. Dias da Costa, R.C. Picão, V.B.D.C. Girão, G. Santoro-Lopes, et al. 2021. Acquisition of antimicrobial resistance determinants in Enterobacterales by international travelers from a large urban setting in Brazil. Travel Medicine and Infectious Disease 41: 102028. https://doi.org/10.1016/j.tmaid.2021.102028.

van Boeckel, T.P., E.E. Glennon, D. Chen, M. Gilbert, T.P. Robinson, B.T. Grenfell, S.A Levin, S. Bonhoeffer, and R. Laxminarayan. 2017. Reducing antimicrobial use in food animals. Science 357 (6358): 1350-1352. https://doi.org/10.1126/ science.aao1495.

Wang, C.C., Y. Feng, L.N. Liu, L. Wei, M. Kang, and Z.Y. Zong. 2020a. Identification of novel mobile colistin resistance gene mcr-10. Emerging Microbes \& Infections 9 (1): 508-516. https://doi.org/10.1080/22221751.2020.1732231.

Wang, Y., Q.X. Luo, T.T. Xiao, Y.Y. Zhu, and Y.H. Xiao. 2021. Impact of polymyxin resistance on virulence and fitness among clinically important gram-negative bacteria. Engineering. https://doi.org/10.1016/j.eng.2020.11.005.

Wang, Y., C.Y. Xu, R. Zhang, Y.Q. Chen, Y.B. Shen, F.P. Hu, D.J. Liu, J.Y. Lu, Y. Guo, $X$. Xia, et al. 2020b. Changes in colistin resistance and mcr-1 abundance in Escherichia coli of animal and human origins following the ban of colistinpositive additives in China: an epidemiological comparative study. The Lancet Infectious Diseases 20 (10): 1161-1171. https://doi.org/10.1016/S1473-3099(2 0)30149-3.

Wirth, T., D. Falush, R.T. Lan, F. Colles, P. Mensa, L.H. Wieler, H. Karch, P.R. Reeves, M.C.J. Maiden, H. Ochman, et al. 2006. Sex and virulence in Escherichia coli: an evolutionary perspective. Molecular Microbiology 60 (5): 1136-1151. https://doi.org/10.1111/j.1365-2958.2006.05172.x.

Wu, L.J., J. Chen, L. Wang, and Z. Wu. 2018. Whole genome sequence of an MCR1-carrying, extended-spectrum $\beta$-lactamase (ESBL)-producing Escherichia coli ST746 isolate recovered from a community-acquired urinary tract infection. Journal of Global Antimicrobial Resistance 13: 171-173. https://doi.org/10.101 6/j.jgar.2018.03.014.

Yi, L.X., Y.Y. Liu, R.J. Wu, Z.S. Liang, and J.H. Liu. 2017. Research progress on the plasmid-mediated colistin resistance gene mcr-1. Yi Chuan 39 (2): 110-126. https://doi.org/10.16288/j.yczz.16-331.

Yuan, J.Z., X.Y. Wang, D.L. Shi, Q. Ge, X.X. Song, W. Hu, D.Y. Wei, C.L. Ge, X. Li, and C.H. Hu. 2021. Extensive antimicrobial resistance and plasmid-carrying resistance genes in mcr-1-positive E. coli sampled in swine, in Guangxi, South China. BMC Veterinary Research 17 (1): 1-10. https://doi.org/10.1186/s12917021-02758-4

Zając, M., P. Sztromwasser, V. Bortolaia, P. Leekitcharoenphon, L.M. Cavaco, A. Ziętek-Barszcz, R.S. Hendriksen, and D. Wasyl. 2019. Occurrence and characterization of mcr-1-positive Escherichia coli isolated from foodproducing animals in Poland, 2011-2016. Frontiers in Microbiology 10: 1753. https://doi.org/10.3389/fmicb.2019.01753.

Zelendova, M., C.C. Papagiannitsis, A. Valcek, M. Medvecky, I. Bitar, J. Hrabak, T. Gelbicova, A. Barakova, I. Kutilova, R. Karpiskova, et al. 2020. Characterization of the complete nucleotide sequences of $\mathrm{mcr}$-1-encoding plasmids from enterobacterales isolates in retailed raw meat products from the Czech Republic. Frontiers in Microbiology 11: 604067. https://doi.org/10.3389/fmicb.2 020.604067.

Zhu, X.Q., Y.Y. Liu, R.J. Wu, H.L. Xun, J. Sun, J. Li, Y.Y. Feng, and J.H. Liu. 2021. Impact of mcr-1 on the development of high level colistin resistance in Klebsiella pneumoniae and Escherichia coli. Frontiers in Microbiology 12: 666782. https://doi.org/10.3389/fmicb.2021.6667.

\section{Publisher's Note}

Springer Nature remains neutral with regard to jurisdictional claims in published maps and institutional affiliations. 